\title{
RADIO LUMINOSITY FUNCTIONS
}

\author{
J.J. CONDON \\ National Radio Astronomy Observatory \\ 520 Edgemont Road, Charlottesville, VA 22903, USA
}

\section{Introduction}

Luminosity functions give the space densities of source populations as functions of spectral luminosity. They constrain source physics, clustering, cosmological evolution, and contributions to the radiation background. Extragalactic radio sources span eight decades of luminosity, so dividing them into luminosity ranges effectively separates them by energy generation mechanism and other fundamental properties. Multivariate luminosity functions divide the source population in additional ways (e.g., by optical morphology, optical absolute magnitude, or radio spectral index) selected to emphasize particular source characteristics.

Traditionally, local radio luminosity functions have been based on optically selected galaxies supplemented at high luminosities by optical identifications from general radio surveys. The galaxies were usually grouped by optical morphology: (1) E and S0 galaxies, thought to contain active galactic nuclei (AGN), and (2) "normal" spiral and irregular galaxies whose radio emission is primarily powered by stars and stellar remnants only. Bivariate luminosity functions related the radio and optical luminosities. For example, Hummel's (1981) luminosity functions of spiral galaxies are based on the assumption that the radio and optical luminosities of normal galaxies should be roughly proportional, and distributions of the ratio $\log (R) \equiv \log [S(\mathrm{mJy})]+\left(B_{T}-12.5\right) / 2.5$ were used to show that barred spirals contain more luminous central sources than do ordinary spirals.

New opportunities arise as more data become available (especially in other wavebands) and new statistical questions about source populations are posed. Many optical selection and classification limitations can be overcome by the use of far-infrared (FIR) data from the IRAS Faint Source Catalog (Moshir et al. 1992). Optical morphology is an imperfect indicator 
of radio source type: Some spiral galaxies contain radio sources powered by AGN, some low-luminosity sources in E/S0 galaxies appear to be powered by stars (Wrobel \& Heeschen 1988), and many radio galaxies have unclassifiable "peculiar" or "disturbed" morphologies. In contrast, the FIR/radio correlation effectively distinguishes nearby normal galaxies (even ultraluminous starbursts are called "normal" galaxies) from genuine AGN with supermassive black holes or other "monsters." An important new application of AGN luminosity functions is testing unified schemes for AGN (Urry \& Padovani 1995, and references therein). Relativistic beaming is most important for compact flat-spectrum $(\alpha \equiv-d \ln S / d \ln \nu<0.5)$ sources, so separate luminosity functions for steep- and flat-spectrum sources are needed. Finally, new radio- and FIR-selected samples of low-redshift $(z<0.1)$ galaxies are large enough to reduce the statistical uncertainties limiting radio luminosity functions of normal galaxies.

\section{The 1.49 GHz Luminosity Function of Normal Galaxies}

The complete sample of 299 spiral and irregular galaxies with $B_{T} \leq+12$, $|b| \geq 15^{\circ}$, and $\delta>-45^{\circ}$ were mapped by the VLA at $1.49 \mathrm{GHz}$. Following Felten's (1977) "standard" procedure, the local group $(D<1.7 \mathrm{Mpc})$ and galaxies within $10^{\circ}$ of the Virgo cluster center were excluded and the $1.49 \mathrm{GHz}$ luminosity function (open circles in Fig. 1) was found by the $\left(1 / V_{\mathrm{m}}\right)$ method (Condon 1989). The small volume in which spiral galaxies brighter than $B_{T}=+12$ can be found limits the statistical usefulness of this sample to $L<10^{22} \mathrm{~W} \mathrm{~Hz}^{-1}$ at $1.49 \mathrm{GHz}$; FIR-selected galaxies are needed to reach higher radio luminosities.

VLA images at 1.49 and $1.425 \mathrm{GHz}$ (Condon et al. 1990, 1995) now cover the IRAS Bright Galaxy Sample (BGS) Parts I (Soifer et al. 1989) and II (Sanders et al. 1995) of sources stronger than $S=5.24 \mathrm{Jy}$ at $\lambda=60 \mu \mathrm{m}$ in the area $\delta \geq-45^{\circ},|b| \geq 10^{\circ}$. Normal galaxies were selected by the requirement $q \equiv \log \left[\left(F I R / 3.75 \times 10^{12}\right) /\left(S_{1.49 \mathrm{GHz}} / 10^{26}\right)\right] \geq+1.85$, where $F I R \equiv 1.26 \times 10^{-14}\left(2.58 S_{60 \mu}+S_{100 \mu}\right)$. The 465 normal galaxies with $D \geq$ $1.7 \mathrm{Mpc}$ and $>10^{\circ}$ from Virgo yield the luminosity function indicated by the filled circles in Fig. 1. The solid curve is not a fit to the radio data. It is the $\lambda=60 \mu$ m luminosity function (Saunders et al. 1990, model 17) with the $\lambda=60 \mu \mathrm{m}$ luminosities converted to $\nu=1.49 \mathrm{GHz}$ by the mean FIR/radio ratio $\left\langle S_{60 \mu} / S_{1.49 \mathrm{GHz}}\right\rangle=121$ of normal galaxies selected at $1.49 \mathrm{GHz}$. The close fit is an immediate consequence of the tight FIR/radio correlation. Ultraluminous galaxies should be slightly radio quiet relative to $\lambda=60 \mu \mathrm{m}$ due to free-free absorption in compact starbursts (Condon et al. 1991c). The broken curve showing the Schechter blue luminosity function (Felten 1977 ) is too steep, so there cannot be a tight blue/radio correlation. 


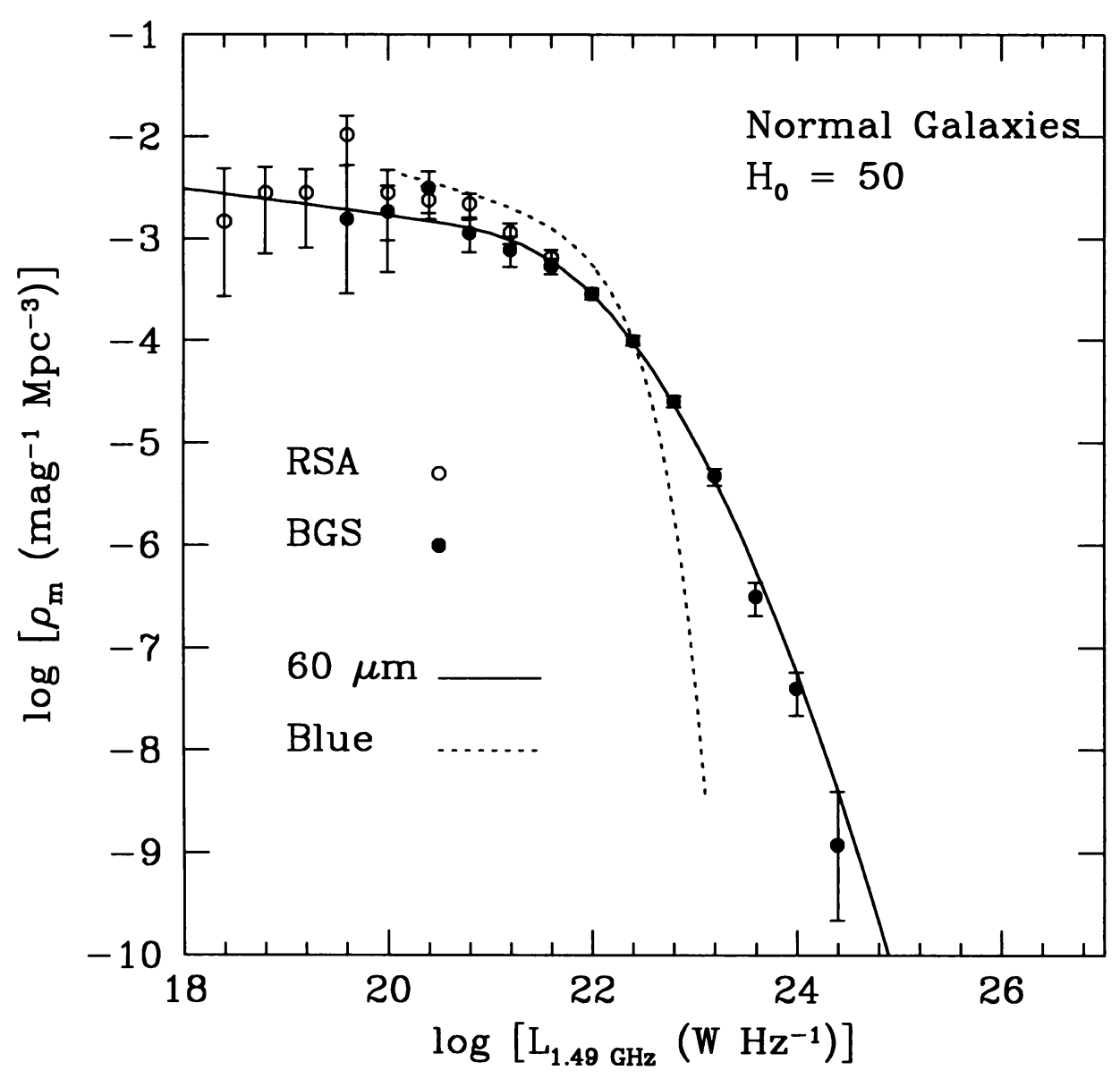

Figure 1. The $1.49 \mathrm{GHz}$ luminosity function of normal galaxies.

This "standard" luminosity function may be high below $\log (L) \approx 21.5$ because the sampled volumes $V_{\mathrm{m}}$ are comparable with the correlation volume of galaxy clustering and are centered on a galaxy-ours. For a twopoint correlation function $\xi(r)$, the mean number $\langle N\rangle_{P}$ of galaxies within a distance $r$ is $\langle N\rangle_{P}=4 \pi r^{3} n / 3+n \int_{0}^{r} \xi(x) 4 \pi x^{2} d x$, where $n$ is the density of galaxies averaged over all space (Peebles 1980, Eq. 31.8). If $\xi(r)=\left(r_{0} / r\right)^{\gamma}$ for $r \leq 2 r_{0}, r_{0} \approx 10.8 \mathrm{Mpc}$, and $\gamma \approx 1.77$ (Davis \& Peebles 1983), then the expected density $\langle n\rangle_{P}$ of galaxies within a distance $r \leq 2 r_{0}$ of our galaxy is $\langle n\rangle_{P} / n=1+3\left(r_{0} / r\right)^{\gamma} /(3-\gamma)$. Thus $V_{\mathrm{m}}$ within $r=2 r_{0}$ should be multiplied by $\langle n\rangle_{P} / n$ to yield luminosity functions representative of all space. The open circles and broken curve in Fig. 2 show the radio luminosity func- 


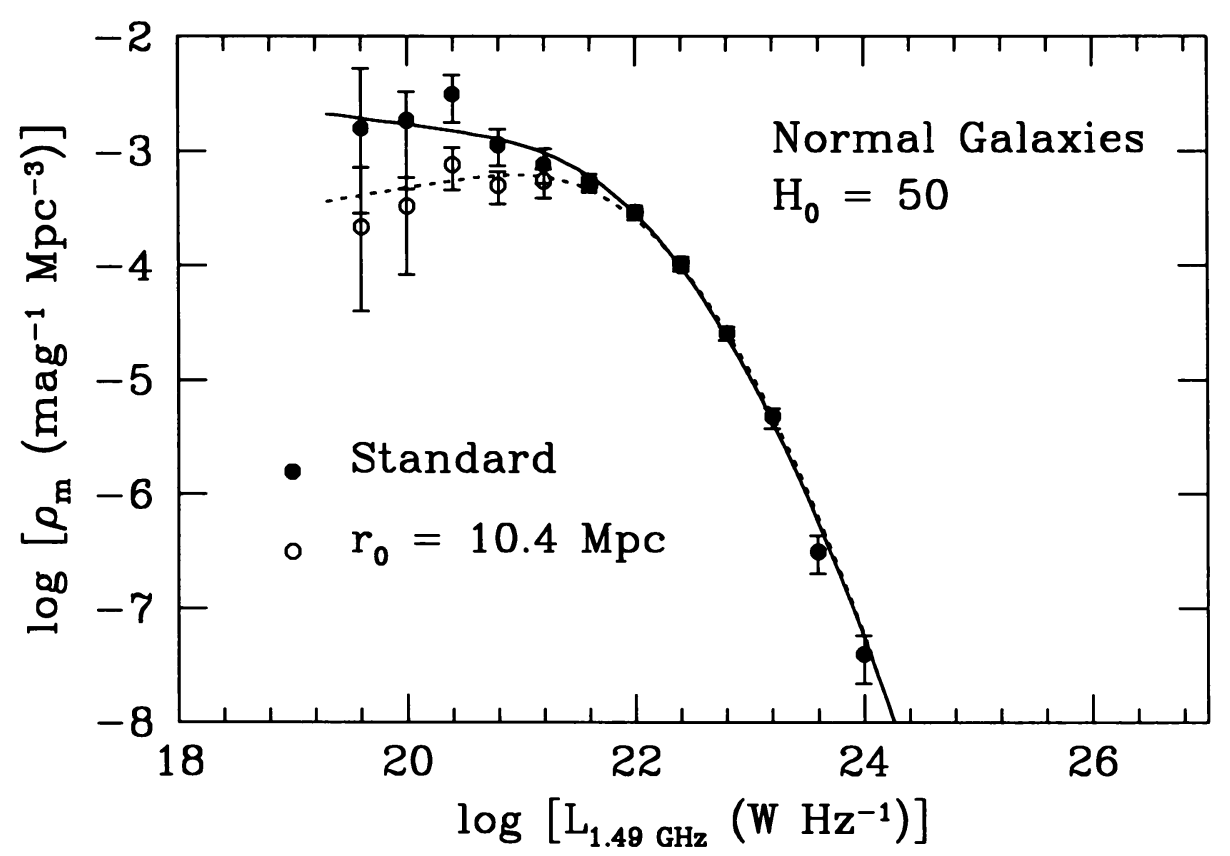

Figure 2. The $1.49 \mathrm{GHz}$ luminosity function of normal galaxies after correction for clustering (broken curve).

tion of 487 BGS galaxies (including those within $D=1.7 \mathrm{Mpc}$ and near Virgo) corrected for the local overdensity. It falls below the $\lambda=60 \mu \mathrm{m}$ luminosity function, in agreement with the nonlinearity of the FIR/radio correlation at low radio luminosities (Condon et al. 1991a). The difference between these two curves is a measure of the uncertainty due to clustering, and it can be reduced only by the use of deeper samples with $V_{\mathrm{m}} \gg r_{0}^{3}$.

\section{The $4.85 \mathrm{GHz}$ Luminosity Function}

A new $4.85 \mathrm{GHz}$ local luminosity function (Fig. 3) was constructed as follows: UGC galaxies with $S \geq 25 \mathrm{mJy}, 5^{\circ}<\delta<+75^{\circ}$, and $|b|>10^{\circ}$ were identified and classified as normal or AGN with the aid of IRAS data (Condon et al. 1991b). Luminosity functions based on 128 normal galaxies and 173 AGN were calculated by the "standard" method described in Sec. 2 and are plotted as filled triangles and circles in Fig. 3. Sixteen nearby $(D<60 \mathrm{Mpc})$ early (E/S0) galaxies brighter than $M_{P}=+14$ and $S=1.5 \mathrm{mJy}^{\text {beam }}{ }^{-1}$ in the $5^{\prime \prime}$ beam of the VLA (Wrobel \& Heeschen 1991) satisfying $u \equiv \log S_{60 \mu} / S_{4.85 \mathrm{GHz}}<2.0$ extended the AGN luminos- 


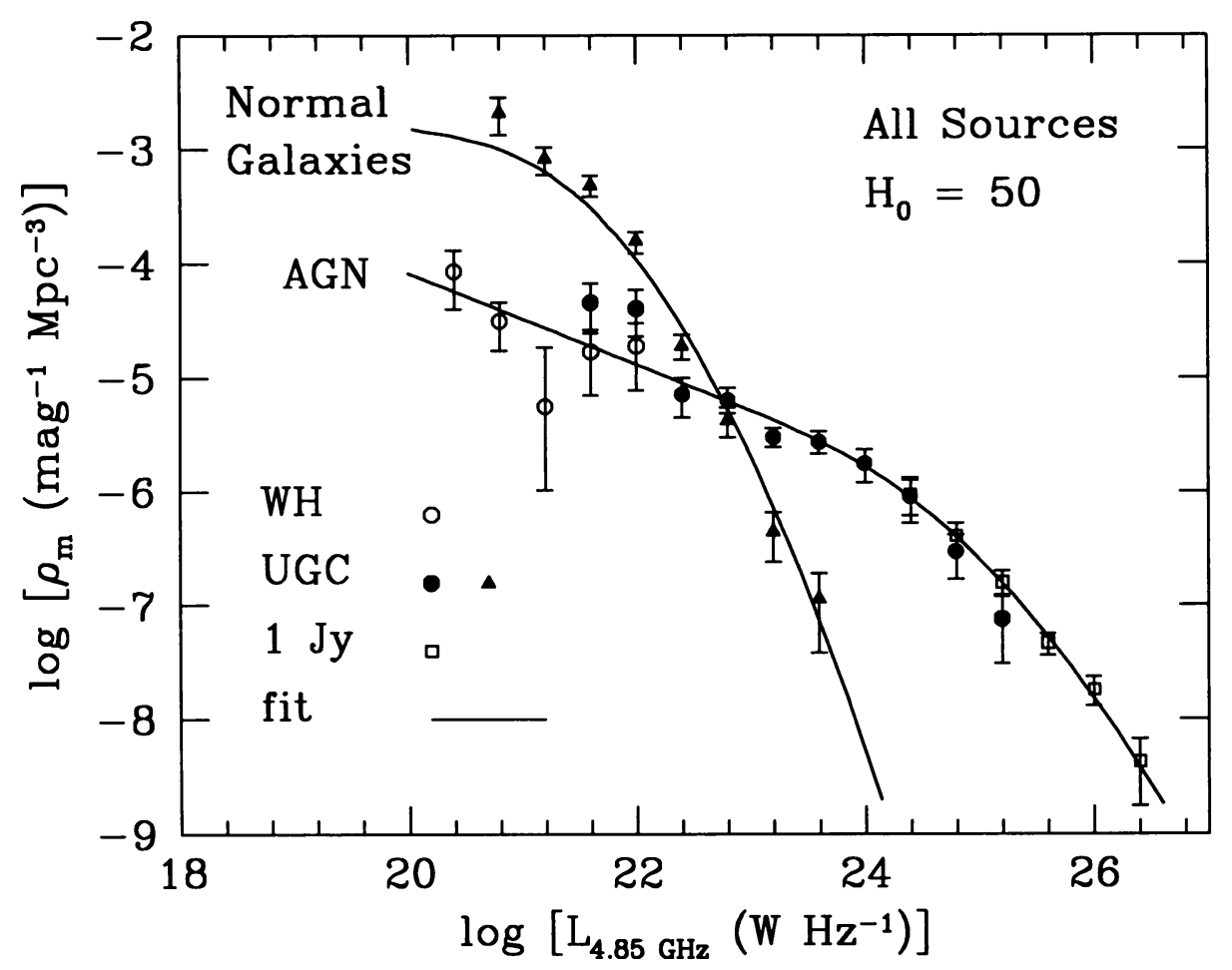

Figure 3. The $4.85 \mathrm{GHz}$ luminosity functions of all radio sources.

ity function as indicated by open circles in Fig. 3. Partial resolution ( $5^{\prime \prime}$ is only $1.5 \mathrm{kpc}$ at $D=60 \mathrm{Mpc}$ ) and clustering both increase the uncertainties at low luminosities. Radio identifications of UGC galaxies with the $1.4 \mathrm{GHz}$ NRAO VLA Sky Survey (NVSS) made with $45^{\prime \prime}$ FWHM resolution should ultimately reduce these uncertainties. Optical identifications of the 83 AGN with $z<0.1$ from the $1 \mathrm{Jy}$ (at $5 \mathrm{GHz}$ ) radio source catalog (Stickel et al. 1994) were added at high luminosities (open squares in Fig. 3).

Figure 4 shows the luminosity function of flat-spectrum $(\alpha<0.5)$ AGN only, along with the fit to all AGN. The fraction of flat-spectrum sources has a weak minimum near the knee at $10^{24} \mathrm{~W} \mathrm{~Hz}^{-1}$. The low-luminosity flat-spectrum sources are primarily in S0 galaxies (Condon et al. 1991b), suggesting an intrinsic difference not attributable to orientation. Matching this luminosity function is a challenge for relativistic beaming models in which high-luminosity sources are the beamed flat-spectrum cores of lowluminosity sources (cf. Orr \& Browne 1982). 


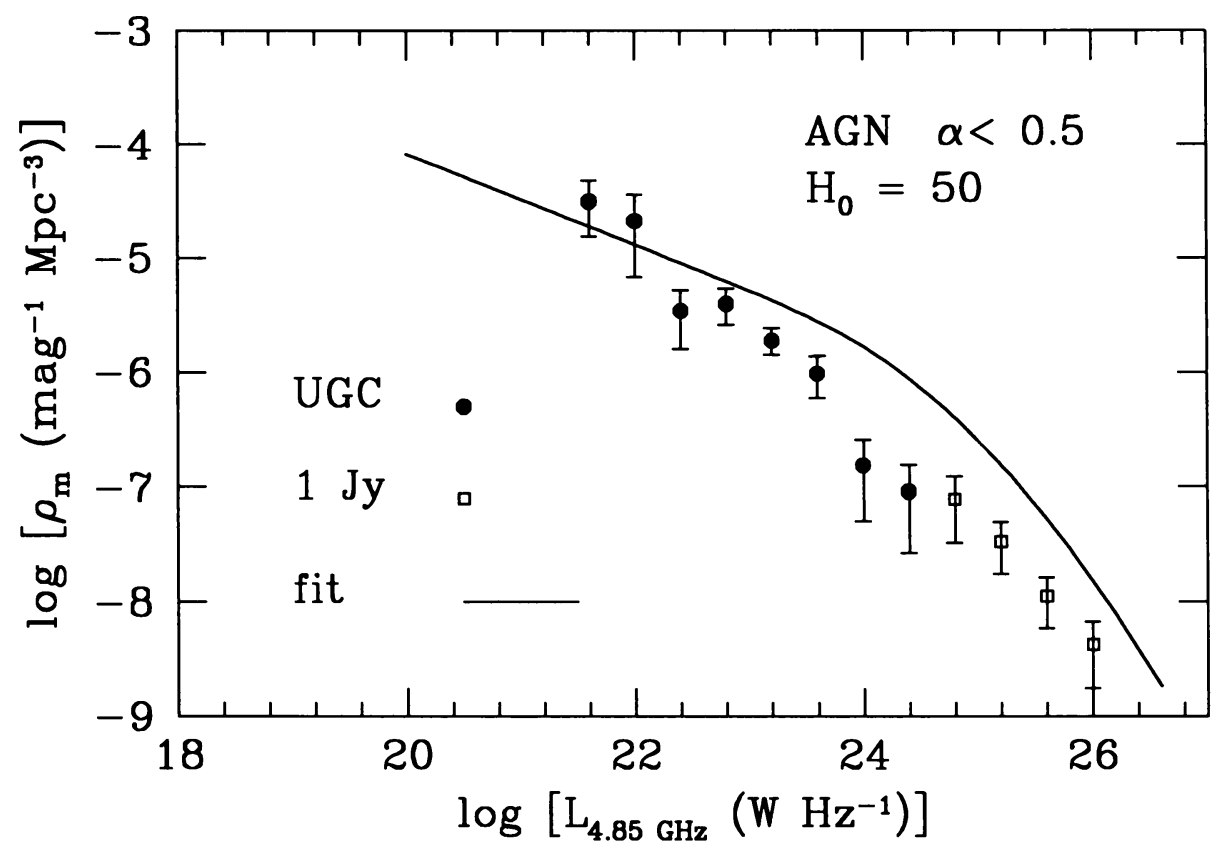

Figure 4. The $4.85 \mathrm{GHz}$ luminosity function of flat-spectrum AGN (data) compared with the that of all AGN (curve).

\section{References}

Condon, J.J. 1989, ApJ, 338, 13

Condon, J.J., Anderson, M.L., \& Helou, G. 1991a, ApJ, 376, 95

Condon, J.J., Frayer, D.T., \& Broderick, J.J. 1991b, $A J, 101,362$

Condon, J.J., Helou, G., Sanders, D.B., \& Soifer, B.T. 1990, ApJS, 73, 359

Condon, J.J., Helou, G., Sanders, D.B., \& Soifer, B.T. 1995, ApJS, in press

Condon, J.J., Huang, Z.-P., Yin, Q.F., \& Thuan, T.X.T. 1991c, ApJ, 378, 65

Davis, M., \& Peebles, P.J.E. 1983, ApJ, 267, 465

Felten, J.E. 1977, $A J, 82,861$

Hummel, E. 1981, $A \& A, 93,93$

Moshir, M., et al. 1992, Explanatory Supplement to the IRAS Faint Source Survey, Version 2, JPL D-10015 8/92 (Jet Propulsion Laboratory, Pasadena)

Orr, M.J.L., \& Browne, I.W.A. 1982, MNRAS, 200, 1067

Peebles, P.J.E. 1980, The Large-Scale Structure of the Universe (Princeton University Press, Princeton)

Sanders, D. B., Egami, E., Lipari, S., Mirabel, I., \& Soifer, B. T. 1995, AJ, in press

Saunders, W., Rowan-Robinson, M., Lawrence, A., Efstathiou, G., Kaiser, N., Ellis, R.S., \& Frenk, C.S. 1990, MNRAS, 242, 318

Soifer, B.T., Boehmer, L., Neugebauer, G., \& Sanders, D.B. 1989, AJ, 98, 766

Stickel, M., Meisenheimer, K., \& Kühr, H. 1994, $A \& A S, 105,211$

Urry, C.M., \& Padovani, P. 1995, PASP, 107, 803

Wrobel, J.M., \& Heeschen, D.S. 1988, ApJ, 335, 677

Wrobel, J.M., \& Heeschen, D.S. 1991, AJ, 101, 148 